\title{
Hybrid Molecular Probe for Nucleic Acid Analysis in Biological Samples
}

\author{
Chaoyong James Yang, Karen Martinez, Hui Lin and Weihong Tan* \\ Center for Research at Bio/Nano Interface, Department of Chemistry and UF Genetics Institute \\ University of Florida, Gainesville, FL32611 \\ RECEIVED DATE (automatically inserted by publisher); tan@chem.ufl.edu
}

\section{Supplemental Information}

DNA Synthesis: All DNA synthesis reagents were from Glen Research (Sterling, VA). All probes and DNAs were synthesized with an ABI3400 DNA/RNA synthesizer (Applied Biosystems, Foster City, CA). FAM CPG and Cy5 phosphoramidite were used for FAM and Cy5 labeling respectively. C18 Spacer phosphamidite was used for the introduction of PEG. Ultra-mild deprotection phosphoramidites were used for probe synthesis, which allowed the probe to be deprotected by overnight incubation in $0.05 \mathrm{M} \mathrm{K}_{2} \mathrm{CO}_{3} /$ methanol. The ultra-mild deprotection avoids degradation of $\mathrm{Cy} 5$ by ammonia in a regular deprotection process. The solutions resulted from deprotection were precipitated in ethanol and the precipitates were then dissolved in $0.5 \mathrm{ml}$ of $0.1 \mathrm{M}$ triethylammonium acetate $(\mathrm{pH} 7.0)$ for further purification with high-pressure liquid chromatography. The HPLC was performed on a ProStar HPLC Station (Varian, CA) equipped with a fluorescent and a photodioarray detector. A C-18 reverse phase column (Alltech, C18, 5U, 250x4.6mm) was used.

Table S1. Sequences of HMP probes and molecular beacon MBTBL and their targets.

\begin{tabular}{ll}
\hline Name & \multicolumn{1}{c}{ Sequence } \\
\hline HMPTBL & Cy5-CTC ATT TTG CTG ATG ACG-(PEG) $)_{\mathrm{n}}$-CTG TCT GGG TAC TCC TCC-FAM \\
Biotinylated HMP & Cy5-CTC ATT TTG CTG ATG ACG-(PEG) -Biotin $_{2}$-(PEG) $)_{8}$-CTG TCT GGG TAC TCC TCC-FAM \\
MBTBL & FAM-CGC ACC TCC TCC CTC TTT TTG CTG GGT GCG-DABCYL \\
Target AT & GCT CAT CAG CAA AAT GAG GGA GGA GTA CCC AGA CAG \\
Target AA & GCT CAT CAG CAA AAA GAG GGA GGA GTA CCC AGA CAG \\
Target AG & GCT CAT CAG CAA AAG GAG GGA GGA GTA CCC AGA CAG \\
Target AC & GCT CAT CAG CAA AAC GAG GGA GGA GTA CCC AGA CAG \\
Random Seq & TCT GTG TAA TCA ACT GGG AGA ATG TAA CTG ACT AGC \\
\hline
\end{tabular}

Optimization of PEG linker length. Four probes, HMPTBL8, HMPTBL12, HMPTBL16 and HMPTBL20, were prepared. The number in the probe name indicates the number of PEG monomer unit in the probe. As shown in Figure S1, when only 8 repeat PEG units were used, as high as 7 fold of signal enhancement was observed. An increase of signal enhancement was observed when the PEG spacer length increased. For probes with short linkers, there are two factors that make short linkers unfavorable for good signal enhancement. First, FRET could occur between the acceptor and donor, which would contribute to background signal. For instance, for HMPTBL8, it had 30 bases and 8 PEG units, the distance between two fluorophores could be as high as $27.6 \mathrm{~nm}$ if the probe was fully stretched. However, the distance was shorter as free probe tended to be in coiled form. Secondly, when the spacer length was short, it hindered those two DNA sequence to hybridize to its target, destabilizing probe-target 
hybrid. On the other hand, when the spacer was too long, HMPTBL20, the probe with 20 PEG units for example, the local concentration of one DNA to the other on the same probe would minimize, lowing the chance of two probes binding to the same target sequence. As a consequence, HMPTBL20 had a slightly lower signal enhancement than HMPTBL16.

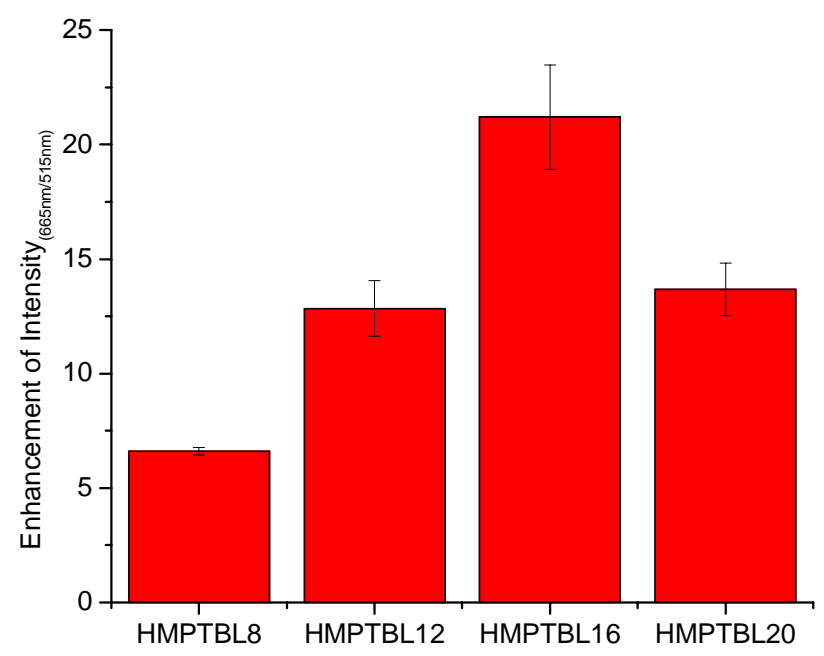

Figure S1. Effect of PEG linker length on the hybridization of linear FRET probe to its target

Acceptor-Donor Distance in Target-Bound probe. The optimization of the AcceptorDonor distance was carried out with a goal to minimize static quenching, while maximizing FRET efficiency. Binding of the probe to its target, as designed, will bring acceptor and donor to close proximity to allow FRET to happen. In the case of FRET, the closer the two fluorophores are, the higher the energy transfer efficiency. However, if two fluorophores get too close to each other, static quenching could happen, which would resulting in quenching of both fluorophores. To get a best signal enhancement, it is important to optimize the distance between these two fluorophores. Different numbers of dTs were inserted into the target DNA sequence to creat various distances between the acceptor and donor after probe-target binding. Table S2 shows the sequences that were used in this experiment. For FAM/CY5 pair, there was no static quenching observed. Instead, the FRET efficiency decreased exponentially with increase FAM/CY5 distance in target/probe hybrid. 


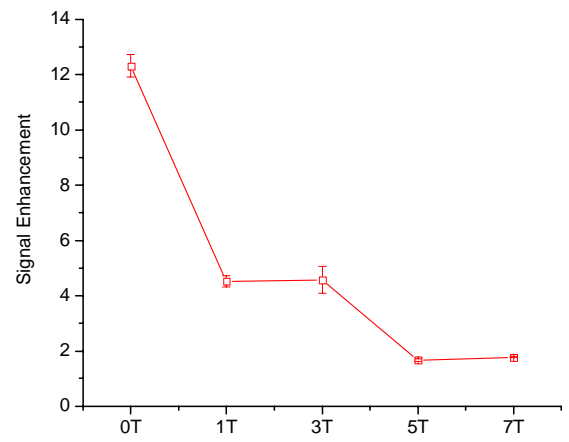

Figure S2. Acceptor-Donor Distance Optimization

Table S2. Target DNA sequences with different numbers of dT

\begin{tabular}{lcc}
\hline Name & \multicolumn{2}{c}{ Sequence } \\
\hline 0T Target & GCT CAT CAG CAA AAT GAG & GGAGGAGTACCCAGACAG \\
1T Target & GCT CAT CAG CAA AAT GAG T & GGAGGAGTACCCAGACAG \\
3T Target & GCT CAT CAG CAA AAT GAG TTT & GGAGGAGTACCCAGACAG \\
5T Target & GCT CAT CAG CAA AAT GAG TTT TT & GGAGGAGTACCCAGACAG \\
7T Target & GCT CAT CAG CAA AAT GAG TTTTTTGGAGGAGTACCCAGACAG \\
\hline
\end{tabular}

HMP for surface DNA hybridization study. One of the advantages of our HMP probe over conventional two-probe FRET system is its larger dynamic range. Another advantage of using HMP is that it can be used for surface hybridization applications such as fiber optic DNA sensors, DNA arrays, and microchannels for nucleic acid detection (Figure S3).

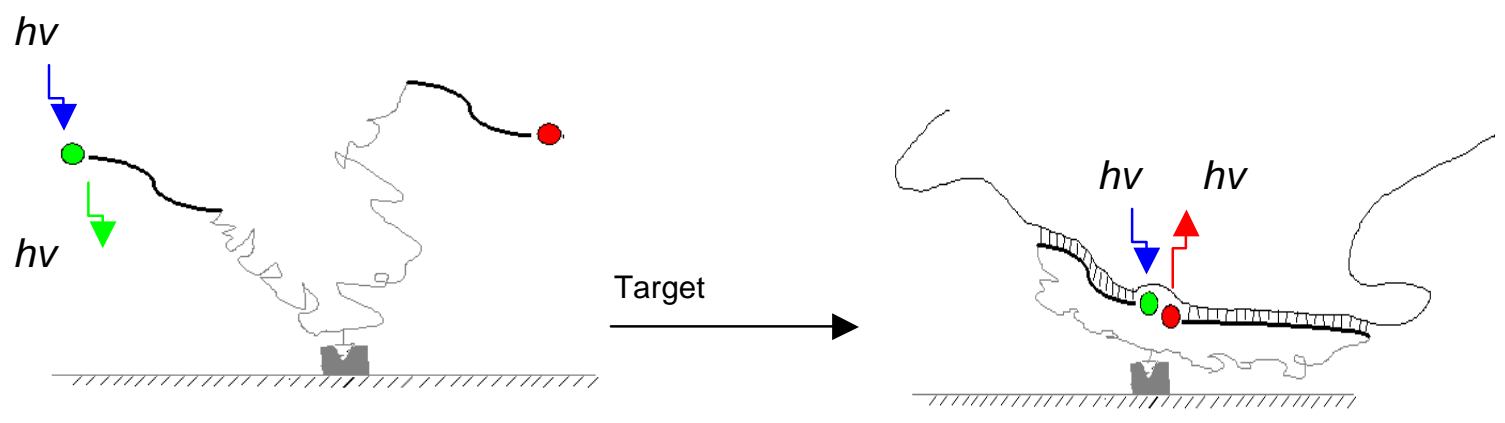

Figure S3. Immobilization of linear DNA FRET probe on solid surface for nucleic acid detection.

A HMP probe was prepared with the same sequence as HMPTBL except that there were two biotins inserted in the middle of linker PEG units. Two biotins were used for one sequence to improve the binding efficiency. Before immobilization onto a streptavidin-coated surface, solution test was performed, which showed similar signal responses of the biotinylated probe to that of the probe without biotin. This indicated that biotin in between the linker did not interfere with the binding of probe to its target. Figure S4 is the response of the immobilized probe upon the addition of target DNA. The surface was excited at $488 \mathrm{~nm}$, and the images were monitored at two emission channels specific for FAM and Cy5 respectively. Fluorescence imaging was 
conducted with a confocal microscope setup consisting of an Olympus IX-81 inverted microscope with an Olympus Fluoview 500 confocal scanning system. Before the hybridization, fluorescence signal from FAM was strong and weak emission from Cy5 was observed. Immediately after addition of cDNA, the intensity of FAM diminished and the intensity of Cy5 increased as a result of hybridization. Overall, fluorescent intensity ratio of Cy5/FAM increased dramatically. From intensity result, large fluctuation for both Cy5 and FAM was observed. This fluctuation was a result of disturbance of the detection system. By taking ratiometric, these noises were cancelled out, and smooth hybridization result was observed. With ratiometric measurement capability, the new DNA probe design removes the internal fluctuations of the detection system, allowing a more precise detection.

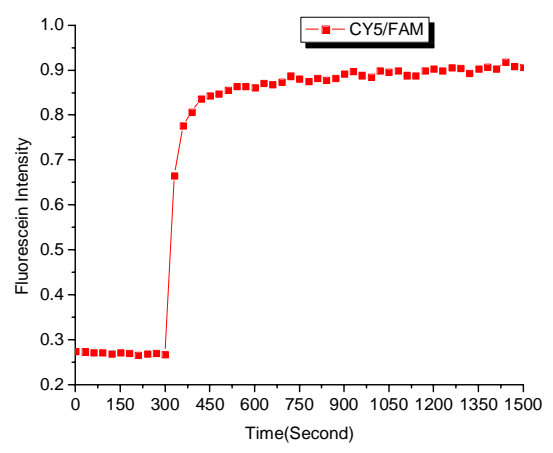

Figure S4 Hybridization of surface immobilized HMP to its target DNA. Buffer: 20mM Tris- $\mathrm{HCl}(\mathrm{pH} 7.5), 50 \mathrm{mM}$ $\mathrm{NaCl}, 5 \mathrm{mM}$ of $\mathrm{MgCl}_{2}$.

Comparison of HMP with Molecular Beacons. A molecular beacon targeting the same target sequence of HMPTBL16 was prepared and used in single-base mismatch detection experiments. Figure S5 shows the response of $300 \mathrm{nM}$ of MBTBL to 300nM of different targets. Under the experiment conditions, perfect matched cDNA was able to produce a signal change of about 8.5 folds. One base mismatch target, while at same concentration, produced less signal change. When $\mathrm{T}$ was changed to $\mathrm{G}$, target DNA changed signal about 4.5 folds. With A replacing $\mathrm{T}$ in the target sequence, target DNA produced about 5.5 times of signal change. Target DNA with $\mathrm{C}$ replacing $\mathrm{T}$, produced only about 2.5 times of change. In case of HMP, it generated about 20 times of signal changed upon hybridized to perfect matched target DNA. While one base mismatch, AT, AC, and AG targets were able to lead to about 12.5,11,14 fold of signal change, respectively. 

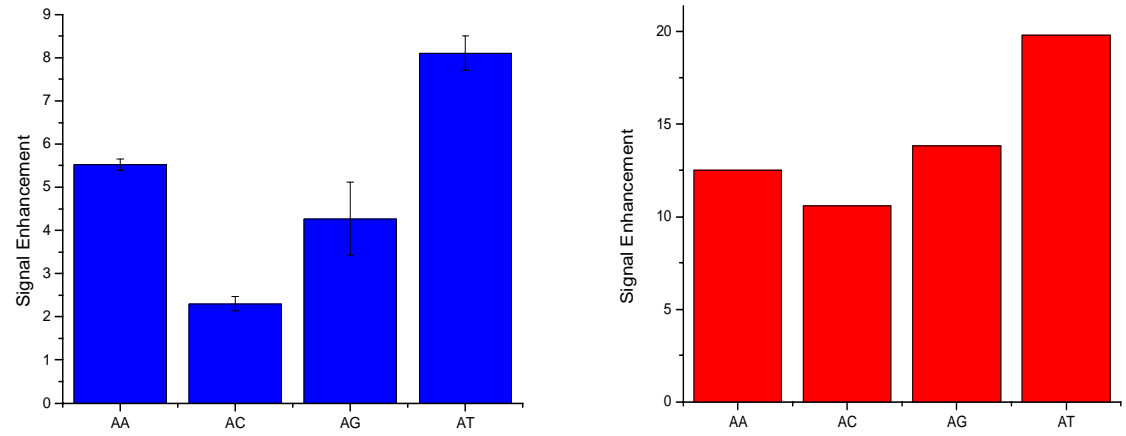

Figure S5 Hybridization result of 300nM of MBTBL(left) and 300nM HMPTBL16 (right) to same concentration of targets. AT stands for the perfect matched target. Buffer: $16 \mathrm{mM}$ Tis- $\mathrm{HCl}(\mathrm{pH} 7.5)$, 40mM NaCl, 4mM $\mathrm{MgCl}_{2}$ and $20 \%$ DMF. 